\title{
Microsurgical carpal tunnel release
}

\section{Scott Shapiro, M.D.}

Section of Neurosurgery, Indiana University Medical Center, Indianapolis, Indiana

The author reports on a series of 482 patients who underwent primary carpal tunnel release (CTR) surgery beginning in 1987. Two hundred twenty-five patients underwent CTR in the right hand, 169 patients underwent CTR in the left hand, and 88 patients underwent bilateral CTR surgery, for a total of 570 hands. These surgeries were performed on an outpatient basis. After application of a local anesthetic, a microsurgical technique using a 2-cm longitudinal incision that did not cross the wrist flexion crease was accomplished. In five patients the recurrent thenar branch exited through the middle or ulnar portion of the ligament and this branch was preserved in all five. Perioperative complications included stitch abscesses in nine hands (2\%), which were managed on an outpatient basis; deep wound infections occurred in three hands $(0.5 \%)$ and these patients were admitted for intravenous antibiotic administration and wound care. One steroid-dependent patient suffered wound dehiscence due to a fall and the wound was primarily repaired. One patient required a second minor procedure to remove a retained suture. Two patients developed "trigger finger" 6 months postoperatively. The mean time until return to work for those patients not receiving Workers' Compensation was 3 weeks and for those receiving Workers' Compensation it was 6.5 weeks. Complete disappearance of painful dysesthesias occurred in 431 (89\%) of 482 patients. Marked improvement occurred in another $33(7 \%)$ patients, yielding an overall improvement rate of $96 \%$. Motor improvement was noted in $438(96 \%)$ of 454 patients with preoperative motor weakness. Bilateral symptoms resolved on the contralateral side following unilateral surgery in seven patients. Fifteen patients (12 of whom were receiving Workers' Compensation) experienced persistent dysesthesias and/or incision pain and did not return to work. An additional eight procedures were performed in patients who had previously undergone surgery at another institution; four showed no evidence of their transverse carpal ligament being sectioned. Five of the eight patients undergoing reexploration noted postoperative improvement in their symptoms. Finally, 14 (3\%) patients presented 1 to 5 years postsurgery with thenar/hypothenar pain. These patients have responded to local steroid injections and are being followed by a hand surgeon.

The reimbursement of the surgeon's fee is $\$ 253.00$ from Medicaid, $\$ 360.00$ from Medicare, and $\$ 560.00$ from preferred provider insurance. The mean total time in the outpatient operating room is 35 to 40 minutes. The outcome and time to return to work are equal, if not superior, to those reported for endoscopic CTR surgery. Microsurgical CTR is thought to be safer and probably more cost-effective.

Key Words * carpal tunnel syndrome * carpal tunnel release * median nerve entrapment * transverse carpal ligament

Surgical division of the transverse carpal ligament (TCL) has been performed since 1933 and is usually a 
relatively simple procedure.[28] A recent report on a microsurgical carpal tunnel release (CTR) technique, performed on an outpatient basis, documented excellent results that were equal to those obtained by using minimally invasive techniques such as the retinaculotome or the endoscope.[26] The reported advantage of the minimally invasive techniques is a small incision, which allows for a short operating time and an early return to work for the patient. The incision used in microsurgical CTR is 2 $\mathrm{cm}$ long and may be shorter in thin patients. An update on this microsurgical approach is reported in a series of 482 patients, with emphasis on duration of the surgical procedure, outcome, complications, and cost.

\section{CLINICAL MATERIAL AND METHODS}

Beginning in 1987, 482 consecutive patients underwent CTR surgery at the Indiana University School of Medicine. Preoperative electromyographic and nerve conduction studies were obtained in all but six patients and only eight $(2 \%)$ showed no evidence for median nerve entrapment at the TCL. Two hundred twenty-five patients underwent CTR surgery in the right hand, 169 in the left hand, and 88 patients underwent bilateral CTR surgery, for a total of 570 hands. Of the 88 patients with bilateral carpal tunnel syndrome (CTS), 18 underwent surgery on both hands during one sitting and 70 underwent surgery on one hand at a time, separated by intervals ranging from 2 to 100 weeks. Many of the patients with bilateral CTS first underwent CTR surgery on the predominantly symptomatic hand and later developed increasing symptoms in the untreated hand. Only in the patients with severe bilateral symptoms did we perform both CTRs at the same time. Motor weakness was determined for the abductor pollicis brevis muscle by direct confrontation testing during the physical examination. 


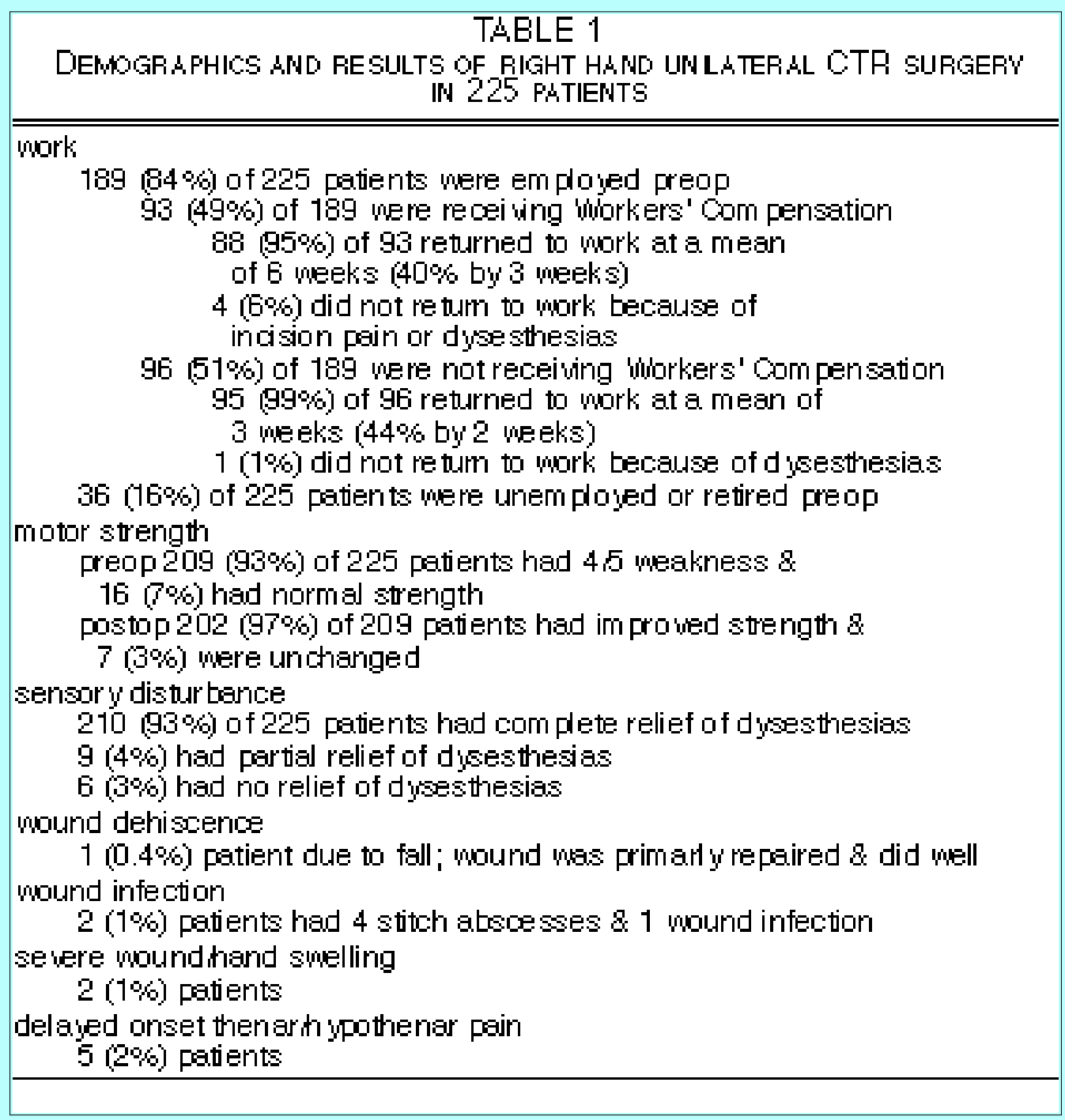

Each patient was offered the choice of receiving local anesthesia or intravenous sedation. Only two patients required a general anesthetic, because they did not comply with constraints surrounding the use of local anesthesia. The local anesthetic was composed of $0.5 \%$ lidocaine in a ration of 1:200,000 with epinephrine. No tourniquet was used. The incision was marked on the ulnar aspect of the thenar crease, just distal to the wrist flexion crease, and extended 2 to $3 \mathrm{~cm}$. Surgery was performed with the aid of either a microscope or surgical loupes. Retraction was maintained by means of mastoid retractors, and hemostasis was attained with bipolar cautery. Blunt and sharp dissection were used to expose the TCL. Because the skin incision was short, hand-held retractors (Senn; Codman, Randolph, MA) were used to increase visualization. The TCL was opened sharply with a 15-blade knife and transected completely with iris scissors. A fine dissector and the iris scissors were used to open the thin translucent ventral epineurium longitudinally over the compressed portion of the nerve. The wound was irrigated with bacitracin and sutured closed with 4-0 ethilon, interrupted, vertical mattress sutures. 


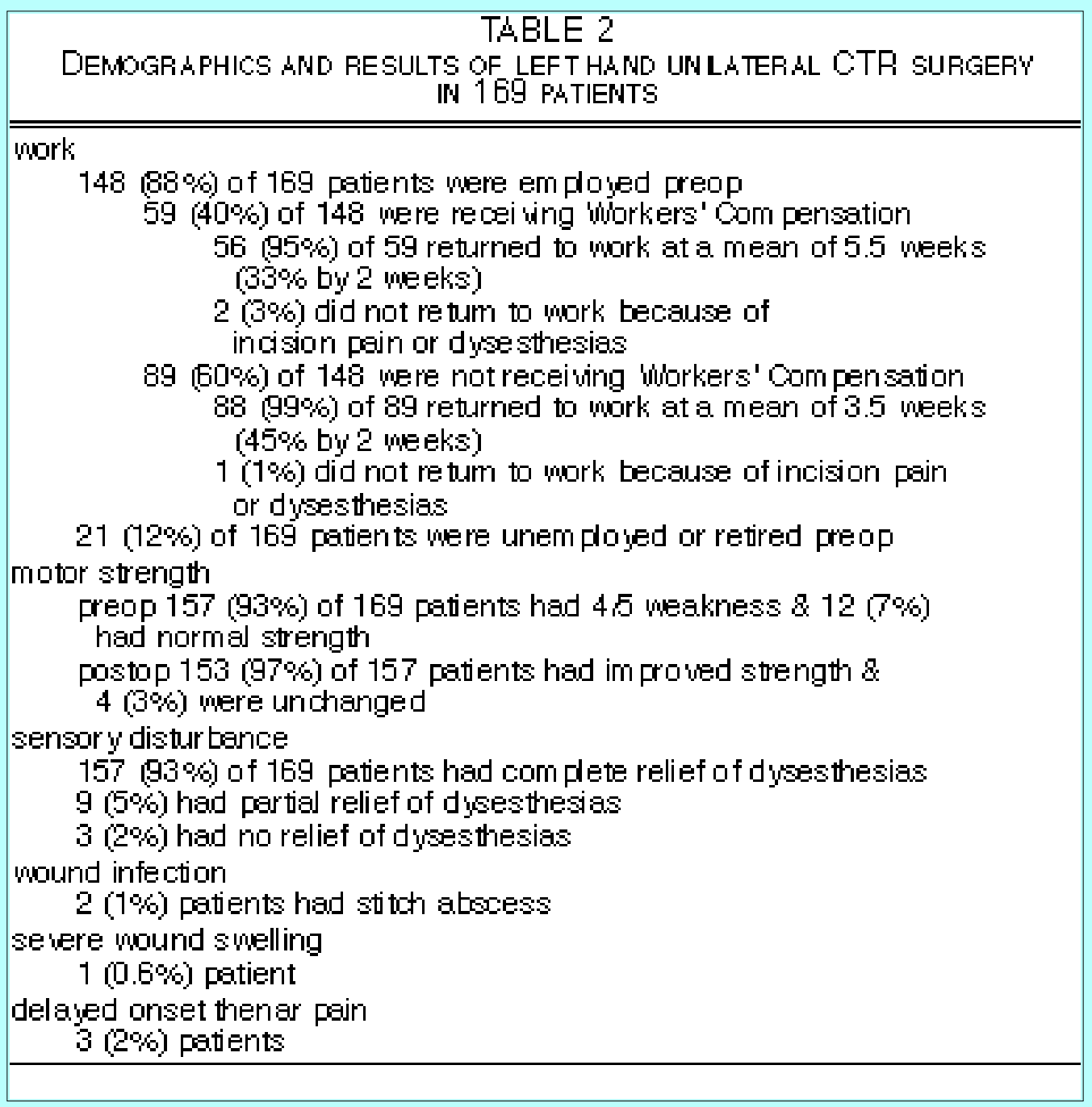

\section{RESULTS}

Tables 1, 2, and 3 show the outcome results for the unilateral and bilateral surgery groups. The only difference observed was a lesser chance of complete relief of dysesthesias in the group in whom bilateral CTR was performed. Fourteen patients (3\%) presented again 1 to 5 years postsurgery suffering from thenar/hypothenar pain. These patients responded to local steroid injection and are being followed by a hand surgeon. Table 4 shows the results of reoperations performed in eight patients in whom symptoms persisted. These patients had previously undergone surgery for CTS at other institutions. Table 5 shows the mean duration and cost of surgery. 


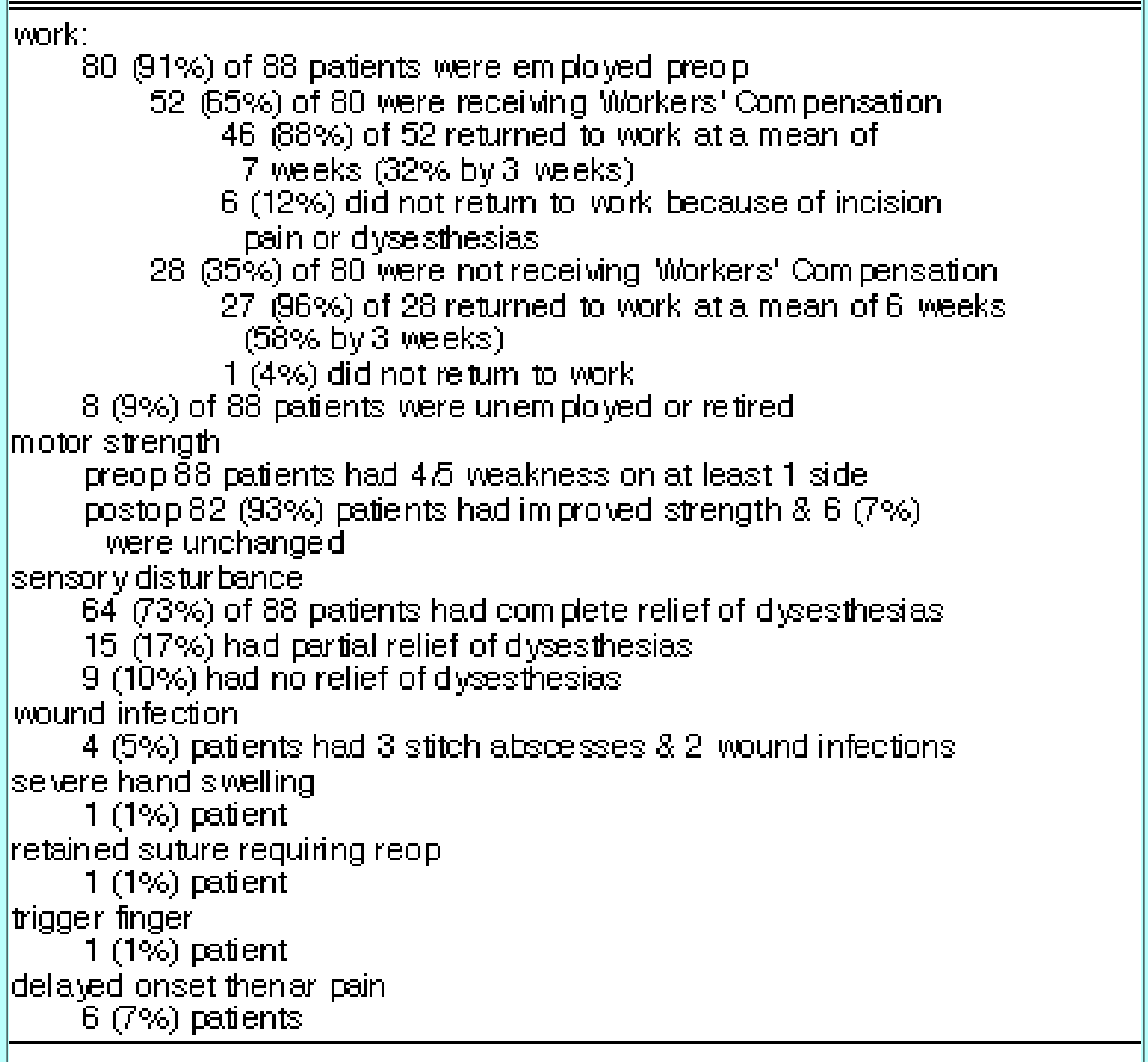

\section{DISCUSSION}

Phalen[19,20] stated that the type of incision used in CTR surgery is of little importance provided that it does not cross the distal flexion crease of the wrist at a right angle. Phalen[18] reported a 99\% improvement in sensory symptoms in 215 patients treated surgically for CTS. Rengachary[21] believed that the incision should be made from the wrist flexion crease extending curvilinearly to a point in line with the distal border of the fully extended thumb. He also noted that, when possible, the proximal incision should not cross the wrist flexion crease, thus preventing injury to the palmar cutaneous nerve. Our minor modification to the technique was to limit the placement of the incision to just distal of the wrist flexion crease and to shorten it to $2 \mathrm{~cm}$. The subcutaneous dissection is easy and, with retraction, all of the ligament can be visualized. Our results demonstrated complete relief of sensory dysesthesias in $431(89 \%)$ of 482 patients; an additional 33 patients (7\%) improved but still experienced some dysesthesias. Motor improvement occurred in $96 \%$ of patients, and there was no motor nerve injury. Numerous reports have documented that sectioning of the TCL by any method leads to excellent results in patients with CTS.[1,5,7,8,17,26,27] 


\section{TABLE 4}

RESULTS OF REOPERATION IN EIGHT PATIENTS UNDERGONG CTR SURGERY*

The TCL had not been sectioned in 4 patients (50\%); all improved after surgery\& 2 retumed to work

The TCL had been sectioned in 4 patients (50\%); no Z-plasty was performed; onl $y$ 1 of 4 im pro ied $\& 2$ of 4 retumed to work

"The first surgeries in these patients were performed by surgeons at other institutions.

Previous reports have described endoscopic CTR (ECTR) surgery as a technically demanding procedure with a significant learning curve. $[2,6,7,9,15,22,23]$ The immediate question that arises is: why make a simple surgery more difficult? One reported advantage for ECTR sugery is a shorter operating time. Our mean operating time is 20 minutes. A postgraduate Year 1 to 3 neurosurgery resident assists with every case and learns the surgical technique and its principles. A report on a technique that required a short open incision documented a mean operating time of 7 minutes for CTR.[16] There is no report showing that ECTR surgery can be performed more quickly than open CTR surgery. There is usually a fixed cost for the ambulatory surgery operating room, which is not dependent on the duration of the procedure. The cost of the anesthetic may, at best, be reduced by one unit if CTR surgery can be performed 15 minutes faster than a mean of 20 minutes (Table 5). Thus, a short operating time will lead to minimal cost savings.

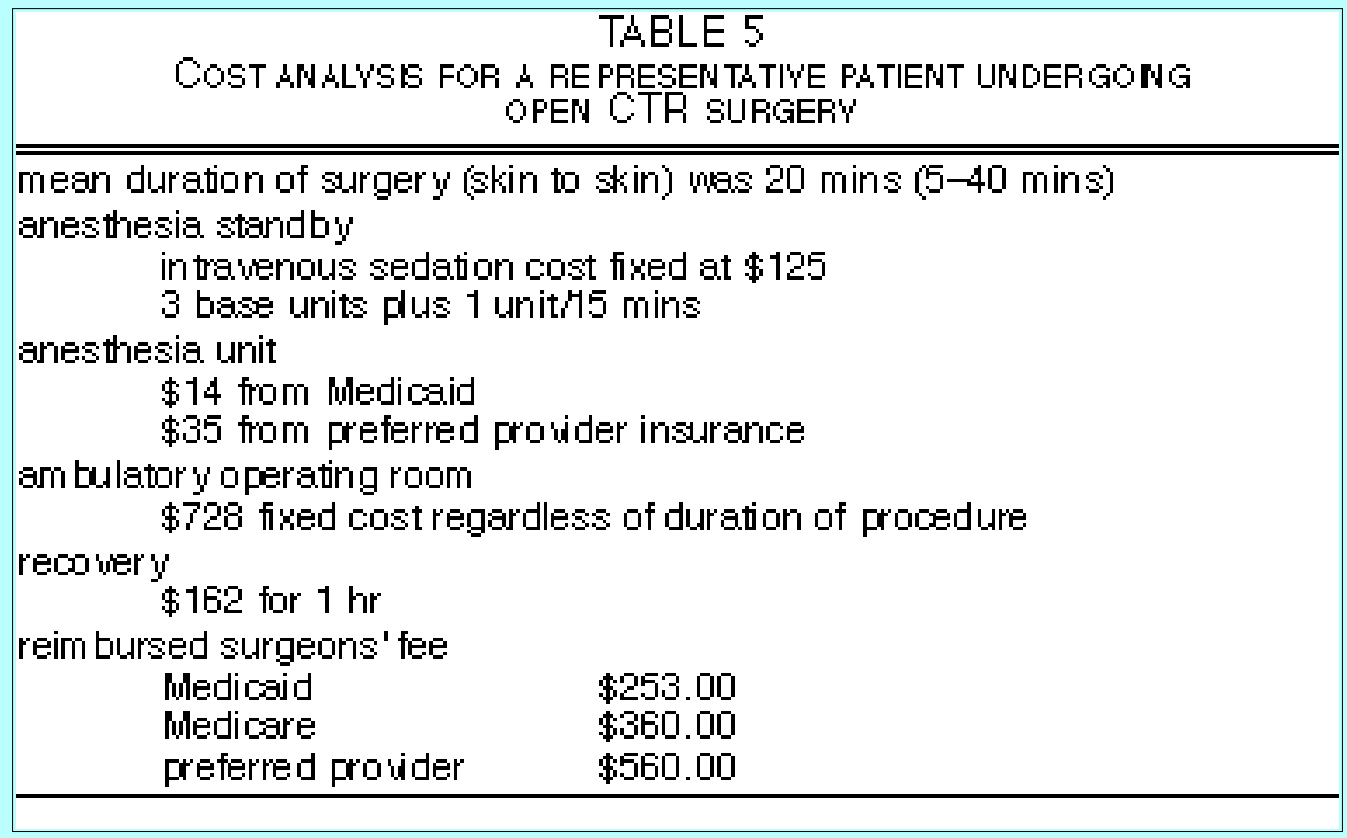

It is difficult to quantitate the effect of a smaller incision on return to work. The biportal technique, which allows direct visualization of the TCL has become a widely used endoscopic procedure, with some reports documenting patients being able to return to work earlier.[23,24] The combined length of the two incisions required for the biportal technique is nearly identical to the 2-cm open CTR technique incision. In a report on 666 ECTR surgeries, the mean time to return to work for patients not receiving Workers' Compensation was 23 days and for those receiving Workers' Compensation it was 60 days; these results are quite similar to ours.[7] A prospective study comparing a minimal-incision open CTR technique to the two-portal ECTR technique documented no significant difference in scar length, need for hand therapy, rate of complications, duration of time before the patient resumed routine activities, duration of time before the patient returned to work, or overall outcome.[9] It has also been documented that early 
hand therapy is more important than incision length in minimizing the interval to regaining normal function and return to work.[16] In fact, early return to work is less dependent on the technique than it is on the motivation of the patient and the type of job the patient performs. We have returned patients to work as soon as 24 hours postsurgery. Other reports have failed to show a significant benefit of ECTR surgery in early return to work analysis.[22,23]

A disturbing and common theme, both in our experience and in the literature, is symptoms persisting in patients who had previously undergone CTR surgery and the discovery at reoperation that the TCL had not been transected.[3,4,11-14] In our experience, most of these operations were performed by physicians who had no formal training in peripheral nerve surgery. Reports on surgeries by means of the ECTR and retinaculotome techniques documented a $0.4 \%$ rate of incomplete TCL transection.[7,17] There is little justification for not sectioning the entire TCL at the first operation.

Although Pagnanelli and Barrer[17] reported no recurrent thenar branch injuries after CTR surgery via a blind technique using the retinaculotome, we remain concerned that the rare aberrant course of the recurrent thenar motor branch can predispose it to be injured in any blind procedure. Median and ulnar nerve transection injuries along with nerve contusion and vascular injuries with both the uniportal and two-portal ECTR techniques have been reported.[3,6,7,15,22,23] One of the problems with the use of an endoscope in CTR procedures is that there is no natural cavity in which the equipment can be inserted, in contrast to its use in cranial and thoracic spine procedures. Thus contusion, stretching, and limited visiblity can all contribute to potential neurological complications. The open microsurgical technique effectively diminishes the likelihood of these problems.

Reported infection rates in CTR surgery range from 0.25 to $6 \% .[1,8]$ Hanssen and coworkers[10] reviewed 3620 cases in which the CTR procedure was used and found a deep-infection rate of $0.47 \%$. Our deep-infection rate was three $(0.5 \%)$ of 570 hands. There are no data to support a reduction in the infection rate with ECTR.

The delayed onset of thenar/hypothenar pain that appeared in 3\% of our patients may be secondary to altered biomechanics at the wrist following TCL transection. Rarely, additional surgery on the carpal bones is necessary to treat uncommon conditions such as piso-triquetral pain syndrome.[25] There are no data to date to support the contention that the ECTR technique will lessen this problem.

The overall cost of surgical procedures is gaining increased importance. If ECTR surgery does not return patients to work more quickly and the equipment to perform the operation is more costly, then there may be no net cost savings.

In conclusion there appear to be many compelling reasons to resist the use of the endoscope to perform CTR surgery. The concern is that many neurosurgical colleagues may feel compelled to learn this demanding and increased-risk technique to compete for patients with hand surgeons who use this technique as a marketing tool.

\section{References}

1. Ariyan S, Watson HK: The palmar approach for the visualization and release of the carpal tunnel. An analysis of 429 cases. Plast Reconstr Surg 60:539-547, 1977

2. Brown RA, Gelberman RH, Seiler JG III, et al: Carpal tunnel release. A prospective, randomized 
assessment of open and endoscopic methods. J Bone Joint Surg (Am) 75:1265-1275, 1993

3. Cobb TK, Cooney WP: Significance of incomplete release of the distal portion of the flexor retinaculum. Implications for endoscopic carpal tunnel surgery. J Hand Surg (Br) 19:283-285, 1994

4. Cotton P: Symptoms may return after carpal tunnel surgery. JAMA 265:1922, 1925, 1991

5. Cseuz KA, Thomas JE, Lambert EH, et al: Long-term results of operation for carpal tunnel syndrome. Mayo Clin Proc 41:232-241, 1966

6. De Smet L, Fabry G: Transection of the motor branch of the ulnar nerve as a complication of two-portal endoscopic carpal tunnel release: a case report. J Hand Surg (Am) 20:18-19, 1995

7. Fischer T, Hastings H, Nagle D, et al: A multicenter prospective review of 666 endoscopic carpal tunnel releases using the Chow technique: American Society for Surgery of the Hand Annual Meeting, $p$ 77, 1990 (Abstract)

8. Gainer JV Jr, Nugent GR: Carpal tunnel syndrome: report of 430 operations. South Med J 70:325-358, 1977

9. Hallock GG, Lutz DA: Prospective comparison of minimal incision "open" and two-portal endoscopic carpal tunnel release. Plast Reconstr Surg 96:941-947, 1995

10. Hanssen AD, Amadio PC, DeSilva SP, et al: Deep postoperative wound infection after carpal tunnel release. J Hand Surg (Am) 14:869-873, 1989

11. Langloh ND, Linscheid RL: Recurrent and unrelieved carpal-tunnel syndrome. Clin Orthop 83:41-47, 1972

12. Louis DS, Greene TL, Noellert RC: Complications of carpal tunnel surgery. J Neurosurg 62:352-356, 1985

13. MacDonald RI, Lichtman DM, Hanlon JJ, et al: Complications of surgical release for carpal tunnel syndrome. J Hand Surg 3:70-76, 1978

14. Murphy RX Jr, Chernofsky MA, Osborne MA, et al: Magnetic resonance imaging in the evaluation of persistent carpal tunnel syndrome. J Hand Surg (Am) 18:113-120, 1993

15. Murphy RX Jr, Jennings JF, Wukich DK: Major neurovascular complications of endoscopic carpal tunnel release. J Hand Surg (Am) 19:114-118, 1994

16. Nathan PA, Meadows KD, Keniston RC: Rehabilitation of carpal tunnel surgery patients using a short surgical incision and an early program of physical therapy. J Hand Surg (Am) 18:1044-1050, 1993

17. Pagnanelli DM, Barrer SJ: Carpal tunnel syndrome: surgical treatment using the Paine retinaculatome. J Neurosurg 75:77-81, 1991

18. Phalen G: The carpal tunnel syndrome. Clinical evaluation of 598 hands. Clin Orthop 83:29-40, 1972

19. Phalen G: Reflections on 21 years' experience with the carpal-tunnel syndrome. JAMA 
212:1365-1367, 1970

20. Phalen G: Spontaneous compression of the median nerve at the wrist. JAMA 145:1128-1132, 1951

21. Rengachary SS: Entrapment neuropathies, in Wilkins RH, Rengachary SS (eds): Neurosurgery. New York: McGraw-Hill, 1985, Vol 2, pp 1771-1776

22. Seiler J, Gelberman R, Weiland A, et al: Operative management of open and endoscopic methods of transverse carpal ligament release. American Society for Surgery of the Hand Annual Meeting, p 29, 1992 (Abstract)

23. Sellers DS: Endoscopic carpal tunnel release. Clin Plast Surg 22:775-780, 1995

24. Sennwald GR, Benedetti R: The value of one-portal endoscopic carpal tunnel release: a prospective randomized study. Knee Surg Sports Traumatol Arthrosc 3:113-116, 1995

25. Seradge H, Seradge E: Piso-triquetral pain syndrome after carpal tunnel release. J Hand Surg (Am) 14:858-862, 1989

26. Shapiro S: Microsurgical carpal tunnel release. Neurosurgery 37:66-70, 1995

27. Tountas CP, MacDonald CJ, Meyerhoff JD, et al: Carpal tunnel syndrome. A review of 507 patients. Minn Med 66:479-482, 1983

28. Woltman HW: Neuritis associated with acromegaly. Arch Neurol Psychiatry 45:680-682, 1941

Manuscript received April 11, 1997.

Accepted in final form June 23, 1997.

Address reprint requests to: Scott Shapiro, M.D., East Outpatient Building, Room 323, Wishard Hospital, Indiana University Medical Center, 1001 West 10th Street, Indianapolis, Indiana 46202. 